\title{
Monte Carlo simulation of coagulation in discrete particle-size distributions. Part 1. Brownian motion and fluid shearing
}

\author{
By H. J. PEARSON, I. A. VALIOULIS AND E. J. LIST \\ W. M. Keck Laboratory of Hydraulics and Water Resources, California Institute of \\ Technology, Pasadena, California 91125
}

(Received 31 March 1982 and in revised form 5 November 1983)

\begin{abstract}
A method for the Monte Carlo simulation, by digital computer, of the evolution of a colliding and coagulating population of suspended particles is described. Collision mechanisms studied both separately and in combination are: Brownian motion of the particles, and laminar and isotropic turbulent shearing motions of the suspending fluid. Steady-state distributions are obtained by adding unit-size particles at a constant rate and removing all particles once they reach a preset maximum volume. The resulting size distributions are found to agree with those obtained by dimensional analysis (Hunt 1982).
\end{abstract}

\section{Introduction}

In many fluid systems with a continuous size distribution of suspended particles the primary mechanism for the production of larger particles from smaller particles, over much of the size range, is coagulation, the process of collision and coalescence of particles. These coagulating particles can be solid or liquid, with the suspending medium gaseous or liquid, for example: atmospheric aerosols, cloud water droplets, colloidal suspensions in water or emulsions of one liquid or another. The computations described in this paper are primarily concerned with suspensions of solid particles in water, but the techniques used have general applications.

In describing the dynamics of continuous size distributions it is convenient to introduce the particle-size distribution $n(v)$, defined by

$$
\mathrm{d} N=n(v) \mathrm{d} v,
$$

so that $\mathrm{d} N$ is the number of particles per fluid volume whose sizes (volumes) lie in the range $v$ to $v+\mathrm{d} v$. The collision rate, per unit volume of fluid, of particles of volumes $v_{i}$ and $v_{j}$ is given by the product of their respective concentrations and a collision function $\beta\left(v_{i}, v_{j}\right)$ representing the geometry and dynamics of the collision mechanism, so that

$$
\text { collision rate }=\beta\left(v_{i}, v_{j}\right) n\left(v_{i}\right) n\left(v_{j}\right) \mathrm{d} v_{i} \mathrm{~d} v_{j} .
$$

Then the change with time of the particle-size distribution is given by the general dynamic equation

$$
\begin{aligned}
\frac{\partial n(v)}{\partial t}= & I(v)+\frac{1}{2} \int_{0}^{v} \beta\left(v^{\prime}, v-v^{\prime}\right) n\left(v^{\prime}\right) n\left(v-v^{\prime}\right) \mathrm{d} v^{\prime} \\
& -\int_{0}^{\infty} \beta\left(v, v^{\prime}\right) n(v) n\left(v^{\prime}\right) \mathrm{d} v^{\prime}+S(v) \frac{\partial n(v)}{\partial z} .
\end{aligned}
$$


Here $I(v)$ is a source of particles (through condensation, for example) and $S(v) \partial n / \partial z$ is a particle sink resulting from particles sedimenting in the $z$-direction at their Stokes settling velocity $S(v)$. If we restrict attention to size ranges where the source term is negligible, and to homogeneous situations (so that spatial derivatives may be neglected), then (1) reduces to the coagulation equation

$$
\begin{aligned}
\frac{\partial n(v)}{\partial t}= & \frac{1}{2} \int_{0}^{v} \beta\left(v^{\prime}, v-v^{\prime}\right) n\left(v^{\prime}\right) n\left(v-v^{\prime}\right) \mathrm{d} v^{\prime} \\
& -\int_{0}^{\infty} \beta\left(v, v^{\prime}\right) n(v) n\left(v^{\prime}\right) \mathrm{d} v^{\prime} .
\end{aligned}
$$

The two terms on the right-hand side of (2) represent respectively the rate of gain of particles of volume $v$ by coagulation of pairs of smaller particles, conserving volume, and the loss of particles, $v$, due to their coagulation with particles of all sizes.

In writing (2) it is assumed that the suspension is completely mixed and that correlations between particles induced by the coagulation process can be ignored. For example, as particles of a given size coalesce, a local reduction in their number occurs, so fewer particles of this size remain for further coalescence. Such spatial inhomogeneities, which can be expressed as conditional probabilities for the collision of two particles (Gillespie 1972) are ignored in (2), whose solution thus may not include all possible histories of particle growth.

A variety of techniques have been used to investigate (1) and (2), and an extensive literature has resulted (for a recent account see Pruppacher \& Klett 1978). In this study we present a Monte Carlo method for the direct simulation of the processes of collision and coalescence of particles. Unlike the direct numerical solutions of (2), such as developed by Gelbard, Tambour \& Seinfeld (1980), the technique avoids approximations involved in writing (2). It also does not require analytic forms of the collision functions $\beta$ (except for when Brownian motion is the collision mechanism; see Appendix A). Direct comparison of the results of the Monte Carlo simulation method and numerical integrations of (2) should therefore prove useful. Furthermore, in addition to predicting the average particle-size spectrum the simulation method provides information on higher moments of properties of the suspension, thereby enabling estimates of the uncertainty of the size spectrum.

In order to confirm the appropriateness of the method and verify the techniques used, it was considered sensible first to ignore particle interactions such as those due to hydrodynamic and interfacial forces. Clearly, this is unrealistic and only defensible as a first step toward a more sophisticated model. However, there seemed little point in proceeding to this enhanced model if the simulation method would not reproduce the results of Hunt and Friedlander which were derived in the absence of interparticle forces. A more complex model including also the simulation of differential settling induced collisions is the subject of a subsequent paper.

For particles to coagulate two processes are required: $(a)$ a mechanism to develop relative motion of the particles through the fluid which will bring them into close proximity; and $(b)$ short-range interfacial forces acting between the particles to bring about their coalescence. Relative motion of particles in a fluid can be due to one or a combination of the following:

1. Brownian or thermal motion;

2. laminar or turbulent fluid shear or straining;

3. particle inertia in turbulent flows;

4. differential sedimentation of different-sized particles. 


\begin{tabular}{|c|c|c|c|}
\hline Mechanism & Collision function $\beta$ & Source & $\begin{array}{l}\text { Dimensional } \\
\text { parameter }\end{array}$ \\
\hline Brownian motion & $\frac{2 k T}{3 \mu} \frac{\left(r_{i}+r_{j}\right)^{2}}{r_{i} r_{j}}=4 \pi\left(D_{i}+D_{j}\right)\left(r_{i}+r_{j}\right)$ & $\begin{array}{l}\text { Smoluchowski } \\
\text { (1916) }\end{array}$ & $K_{\mathrm{B}}=\frac{k T}{\mu}$ \\
\hline Laminar shear & $1.33 G\left(r_{i}+r_{j}\right)^{3}$ & $\begin{array}{l}\text { Smoluchowski } \\
(1917)\end{array}$ & $G$ \\
\hline $\begin{array}{l}\text { Pure strain } \\
\text { (extension) }\end{array}$ & $4.89 \dot{\gamma}\left(r_{i}+r_{j}\right)^{3}$ & $\begin{array}{l}\text { Zeichner and } \\
\text { Schowalter } \\
\text { (1977) }\end{array}$ & $\dot{\gamma}$ \\
\hline $\begin{array}{l}\text { Isotropic turbulent } \\
\text { shear }\end{array}$ & $2.3 \dagger\left(r_{i}+r_{j}\right)^{3}(\epsilon / \nu)^{\frac{1}{2}}$ & $\begin{array}{l}\text { Saffman and } \\
\text { Turner (1956) }\end{array}$ & $(\epsilon / \nu)^{\frac{1}{2}}$ \\
\hline Turbulent inertia & $\frac{1.27\left(\rho_{\mathrm{p}}-\rho_{\mathrm{f}}\right)}{\mu}\left(\frac{\epsilon^{3}}{\nu}\right)^{\frac{1}{4}}\left(r_{i}+r_{j}\right)^{2}\left|r_{i}^{2}-r_{j}^{2}\right|$ & $\begin{array}{l}\text { Saffman and } \\
\text { Turner (1956) }\end{array}$ & $\frac{\rho_{\mathrm{p}}-\rho_{\mathrm{f}}}{\mu}\left(\frac{\varepsilon^{3}}{\nu}\right)^{\frac{1}{4}}$ \\
\hline $\begin{array}{l}\text { Differential } \\
\text { sedimentation }\end{array}$ & $\frac{0.7 g\left(\rho_{\mathrm{p}}-\rho_{\mathrm{q}}\right)}{\mu}\left(r_{i}+r_{j}\right)^{2}\left|r_{i}^{2}-r_{j}^{2}\right|$ & $\begin{array}{l}\text { Findheisen } \\
(\mathbf{1 9 3 9 )}\end{array}$ & $K_{\mathrm{ds}}=\frac{g\left(\rho_{\mathrm{p}}-\rho_{\mathrm{f}}\right)}{\mu}$ \\
\hline \multicolumn{4}{|c|}{ † Corrected from original - see text. } \\
\hline
\end{tabular}

If hydrodynamic and interfacial forces between particles are ignored, relatively simple analytic estimates for $\beta$ are available for each of these collision mechanisms acting independently, and these are summarized in table 1 . The table also includes the dimensional parameters that characterize the mechanisms and determine, in any given situation, the characteristic size of particles that they affect.

Note that all the collision functions depend on properties of the suspending fluid, the structure of its velocity field, and the size of the particles. However, only the functions for the final two collision mechanisms depend on a physical property of the particles: the difference between their density and that of the fluid. If the particle density excess ratio $\left(\rho_{\mathrm{p}}-\rho_{\mathrm{f}}\right) / \rho_{\mathrm{f}}$ is small then sedimentation and inertia will only be important for larger particles. In a turbulent flow sedimentation will dominate inertial effects unless the characteristic acceleration $\left(\epsilon^{3} / \nu\right)^{\frac{1}{4}}$ is comparable to $g$, the gravitational acceleration. In this paper we will be concerned only with the first two collision mechanisms.

For a coagulating system more than one collision mechanism can be important for a given size range of particles. However, if there is a particle size subrange in which the coagulation is dominated by only one collision mechanism, and this subrange is in a state of dynamic equilibrium, then the theory of Friedlander $(1960 a, b)$ and Hunt (1982) predicts the local size distribution given a constant flux of mass through the particle-size distribution. Aside from ignoring interparticle forces, the theory depends on two basic hypotheses: an equilibrium size distribution being established and non-interference of particles of a size characteristic of one collision mechanism with those of another collision mechanism.

Hunt's (1982) experimental results generally support the predictions of the theory for Brownian motion and laminar shear but are limited by uncertainty over the effects of the unsteadiness in the experiments due to particle sedimentation and loss from the system. In the present work these limitations are overcome by performing a 
computer 'experiment' in which particle collisions are directly simulated by Monte Carlo techniques. The size evolution of a population of particles is followed. This allows the effects of each collision mechanism to be evaluated independently, and, by combining mechanisms, the hypothesis of non-interference of characteristic particle sizes to be tested. Collision rates as well as the approach to and the final form of an equilibrium size distribution are studied. The method could also be used to study the 'ageing' of an initially fixed number of particles as they collide and grow.

Monte Carlo simulations have been used by Nowakowski \& Sitarski (1981) to model the collision function for Brownian coagulation of aerosol particles and by Husar (1971) and Gartrell \& Friedlander (1975) to find solutions to the coagulation equation (2). In this paper we first briefly describe Hunt's theory and experimental results. Subsequent sections describe in detail the simulation techniques used to model Brownian, laminar-shear- and turbulent-shear-induced coagulation and the results obtained. The results are then compared with previous experiments and theory, and the success of the method evaluated.

\section{Hunt's work}

Friedlander $(1960 a, b)$ explained observed regularities in the size distributions of atmospheric aerosols by assuming that a state of dynamic equilibrium existed between production, coagulation and loss through sedimentation of particles. He then employed methods analogous to those developed by Kolomogorov for the analysis of turbulence spectra. If it is assumed that the size distribution in some subrange depends only on the particle volume $v$, the constant flux $E$ of particle volume through the size distribution, and a dimensional parameter $C$ characterizing the sole dominant coagulation mechanism (see table 1) so that

$$
n(v)=n(v, E, C)
$$

then the form of $n(v)$ can be determined by dimensional analysis alone. This is analogous to postulating an inertial subrange of scales in which the turbulent-energy spectrum is determined solely by the wavenumber and the flux of energy through the subrange (equal to the rate of energy dissipation by viscous stresses at the smallest scales) (see e.g. Monin \& Yaglom 1975, chap. 21).

Hunt (1982) extended these ideas to hydrosols and compared the predictions of his theory with both laboratory and field measurements. In particular, he performed experiments on Brownian and laminar shear-induced coagulation. His theory predicts the following size distributions for regions dominated by Brownian, shear and differential-sedimentation coagulation:

Brownian

$$
n(v)=a_{\mathrm{B}}\left(\frac{E}{K_{\mathrm{B}}}\right)^{\frac{1}{2}} v^{-\frac{3}{2}}
$$

shear

$$
n(v)=a_{\mathrm{sh}}\left(\frac{E}{G}\right)^{\frac{1}{2}} v^{-2}
$$

differential sedimentation

$$
n(v)=a_{\mathrm{ds}}\left(\frac{E}{K_{\mathrm{ds}}}\right)^{\frac{1}{2}} v^{-\frac{13}{6}} .
$$


He shows (Hunt 1982, figure 1) that it is plausible, for a typical coagulating hydrosol, that these three mechanisms could dominate in regions of successively increasing particle size.

Hunt's measurements indicated that this system was in a quasi-dynamic equilibrium where size distributions taken at progressively later times were similar in shape but decreasing in magnitude. This unsteadiness was due to the overall particle concentration decreasing as a result of the larger aggregates settling to the bottom. Hunt measured the varying total suspended volume by light absorbance and used the computed rate of volume loss as an estimate for $E$. He explains why this will be an overestimate for the quantity (for details see Hunt 1982), but it is still a useful approximation. The measured value of $E^{\frac{1}{2}}$ can then be used to normalize size distributions (cf. (3)-(5)) partially correcting for the effects of unsteadiness.

Hunt successfully collapsed much of his data at various times and for different experiments at different shear rates by normalizing the size distributions not just with $E^{\frac{1}{2}}$ but with the ratio $(E / G)^{\frac{1}{2}}$, and non-dimensionalizing the particle volume with the characteristic volume at which particles have both Brownian collisions and shearinduced collisions at the same rate. This characteristic volume, found by putting $r_{i}=r_{j}$ in the expressions for the relevant collision rates in table 1 , is seen to be $v=\pi K_{\mathrm{B}} / 3 G$, proportional to the ratio of the Brownian and shear parameters.

For some of the particle types tested the normalized volume distributions expressed as functions of non-dimensional size provide support for the relations (3) and (4) (see in particular Hunt 1982, figure 5). However, as we have already noted, there are some reservations about the experiments, complicated as they are by instrumental difficulties and uncertainties about the effects of unsteadiness. Also, no one single experiment exhibits a size distribution having regions with the equilibrium power laws corresponding to both Brownian and shear-dominated mechanisms. One of the main aims of the present study, then, is to provide support or otherwise for Hunt's results by means of a computer 'experiment'. This allows a genuine steady state to be set up and detailed probing of the interaction between Brownian and shear collision mechanisms.

\section{Computer simulation}

\subsection{General technique}

The simulation proceeds by tracking the positions and sizes of a variable number $N$ of spherical particles (typically $50<N<600$ ). Whenever two particles collide they are coagulated to form a larger (still spherical) particle, conserving particle volume, thereby reducing $N$ by one. The population of particles studied therefore consists of particles of unit volume $v_{0}$ and integral multiples $v_{i}=i v_{0}$ of the unit volume. In this paper the suffix $i$ is used to denote properties of $i$-fold particles made up from $i$ elemental particles. All lengths and times in the computer model are nondimensionalized with the radius of the unit particle and the time step $\Delta t$. The collision simulation algorithm is programmed for a digital computer.

The program can also function in a different mode in which collisions are counted but particles are not coagulated. On collision, one of the particles is randomly repositioned so as to avoid repeated collisions of the same pair of particles. This allows direct measurement of the collision function $\beta$ for any given mechanism.

Particle motions take place in a cubical box or 'control volume' of side $L$ and volume $V$. (Figure 1 gives a schematic representation of this box and a definition of the rectangular coordinate system used.) Particle positions are denoted by 


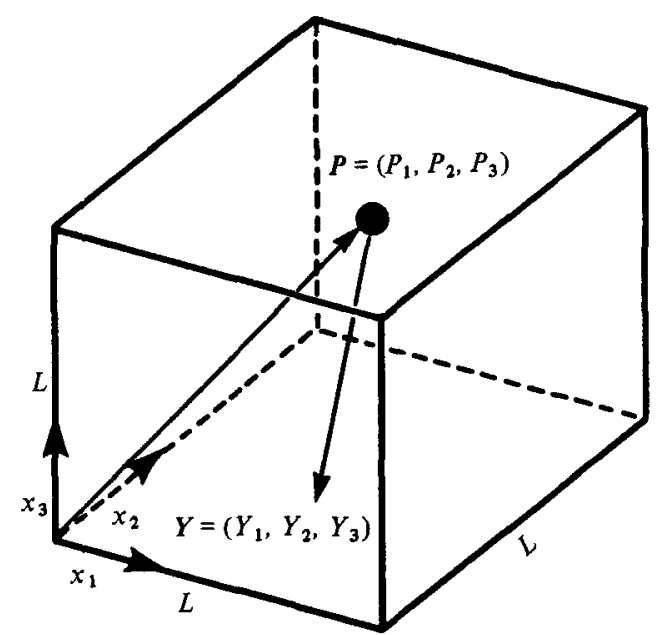

Figure 1. Schematic diagram of simulation box or 'control volume' with Cartesian coordinate system and representative particle at position $\left(P_{1}, P_{2}, P_{3}\right)$. The displacement of the particle in the current time step is $\left(D_{1}, D_{2}, D_{3}\right)$.

$P(i)=\left(P_{1}(i), P_{2}(i), P_{3}(i)\right)$. The simulation employs what are essentially periodic boundary conditions, so that particles that have left the control volume at the end of a time step are replaced, for the next time step, by image particles that enter from the opposite side. This type of boundary condition is commonly employed in Monte Carlo simulations (see Alder \& Wainwright 1969) and allows an infinite homogeneous system to be modelled approximately by a finite volume. Edge effects are reduced by allowing particles to interact with image particles just outside the control volume. The slight modifications to these boundary conditions required for laminar and turbulent shearing motions are described in $\$ \$ 3.4$ and 3.5 below.

In order to model a system in dynamic equilibrium, a fixed number $n_{\mathrm{c}}$ of particles of unit volume are added to the population at random each time step and any particles that have reached a preset maximum volume $v_{\max }=i_{\max } v_{0}$ are removed from the population (typically, $I_{\max }=125$ ). The constant addition of small particles is a crude attempt to represent, indirectly, the particle volume flux $E$ into the size range from the collision of particles smaller than $v_{0}$. In the simulation

$$
E=\frac{v_{0} N_{\mathrm{c}}}{V \Delta t}
$$

The removal of large particles is necessary to limit the total volume density of particles in the simulation. It can be physically justified as a crude representation of the loss of larger particles from a region by the combined action of sedimentation and vertical concentration gradients. The procedure of adding small particles and extracting large ones is consistent with the hypothesis that collisions between particles of similar size are more important, and is justified a posteriori by the success of the simulation in reproducing Hunt's (1982) dimensional results.

The simulation starts either by generating a monodisperse population of particles randomly distributed over the control volume, or by reading a set of particle positions and sizes from a preexisting file. This file is either a set of particles of given size distribution generated by an auxiliary program, or the endpoint of a previous simulation that is to be continued. Controlling parameters for the simulation run are either input manually or read from a file. 
The particular methods for generating the particle displacements at each time step, $Y(i)=\left(Y_{1}(i), Y_{2}(i), Y_{3}(i)\right)$, and updating their positions between time steps are described in detail below in connection with each physical collision mechanism. Each particle is assumed to travel on a straight-line path at constant speed during each time step. The algorithm used to detect particle collisions is described in $\S 3.2$ below.

At the end of every time step the particle-size distribution is computed. After a prescribed number $k$ of time steps, the size distribution, averaged over time $t=k \Delta t$, is placed, along with the positions and sizes of all the particles, in a file in permanent computer storage. The particle positions and sizes are written over the previous copy to save storage space. The latest version is then always available to restart a run at a later time. The simulation continues until the required number of time steps have been completed.

Time averages are needed to provide reasonable particle size statistics as only a small number of particles are followed. Once a simulated system has reached a statistical steady state (dynamical equilibrium) then long-time averages can be employed to produce well-converged statistics. To follow the evolution of a rapidly changing system with any precision, it would be necessary to repeat the simulation many times and compute ensemble averages.

Most simulations were started with a monodisperse population of particles. The total volume of particles in the simulation increases continuously until the first particle grows by coagulation to $v_{\max }$ and is removed. In order to have reasonable computational times the volume concentration $\phi$ of suspended particles used in the simulations is larger than that occurring in many natural systems. (For example, typically $\phi$ is about 20 p.p.m. in Hunt's experiments, but is about $10^{3}$ larger in the simulation runs.)

The simulation requires the generation of relatively large numbers of (pseudo-) random numbers from both uniform and Gaussian distributions. First, a sequence of (pseudo-)random numbers distributed uniformly on the interval $(0,1)$ are generated by the standard congruence method. Random variates are then scaled to any required uniform distribution. Variates with Gaussian distribution are generated from this sequence by various algebraic manipulations and employing a six-constant rational function approximation to the inverse of the Gaussian cumulative distribution function (for details see Abramowitz \& Stegun 1964, \$\$26.2.23 and 26.8).

\subsection{Collision algorithm}

Detecting which particles have collided at each time step is very costly in computer time, and so an efficient method is needed. To this end the basic control volume is divided into cubic subcells. The cells are chosen to be as small as possible consistent with the constraint that any particle can only collide, during the next time step, with particles in the same cell or the adjoining 26 cells. Each cell is given three integer coordinates that define its position in the control volume. For each particle the numbers of the cell it occupies are stored along with its actual position.

The first stage in checking for collisions is to determine for each pair of particles whether they are in the same or adjoining cells. Only if this is so are they considered candidates for a collision and a detailed calculation performed. Checking whether particles are in adjoining cells is performed by computationally fast integer arithmetic. Given two candidate particles, their relative initial position $R P=P(1)-P(2)$ and displacement $R Y=Y(2)-Y(1)$ are computed (note the different ordering of particles). Then the condition for collision is that the vector $R Y$ enters the sphere of radius $\sigma=r_{i}+r_{j}$ around the point $\boldsymbol{R} \boldsymbol{P}$, a simple geometrical test. This corresponds to 

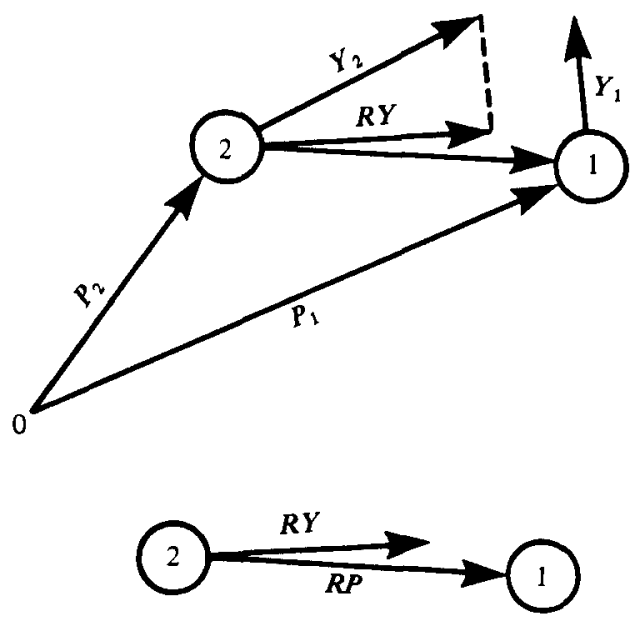

Figure 2. (a) Geometry for collision algorithm. (b) Viewed in frame of reference in which particle 2 is at rest.

following the motion of the two particles in a frame of reference moving with the (1) particle (see figure 2 for schematic illustration).

A further advantage of the subcell system is that it allows for easy implementation of periodic boundary conditions. Particles in cells along any of the boundaries of the control volume are allowed to interact with particles in the requisite cells on the opposite side of the volume.

\subsection{Brownian motion}

The thermal impact of molecules cause suspended particles to perform random motion relative to the bulk fluid. In contrast with the recent work of Nowakowski \& Sitarski (1981), the particles studied here are much larger than the molecular free path in the fluid, and so are in the continuum regime of Brownian motion. Also the time step $\Delta t$ of the simulation is very much larger than the particle viscous relaxation time $t_{\mathrm{r}}=2 r^{2} / 9 v$. Therefore the relevant probability distribution function (p.d.f.) for the displacement $Y$ of a particle during a time step is (Chandrasekhar 1943)

$$
W(Y)=\frac{1}{(4 \pi D \Delta t)^{\frac{3}{2}}} \exp \left(-\frac{|Y|^{2}}{4 D \Delta t}\right)
$$

Where $D$ is the diffusivity of the particle:

$$
D=\frac{k T}{6 \pi \mu r}=\frac{K_{\mathrm{B}}}{6 \pi r} .
$$

Each component of $\boldsymbol{Y}$ has an independent Gaussian p.d.f.

$$
W\left(Y_{k}\right)=\frac{1}{(4 \pi D \Delta t)^{\frac{1}{2}}} \exp \left(-\frac{Y_{k}^{2}}{4 D \Delta t}\right) \quad(k=1,2,3),
$$

and this is used to replace the Brownian motion of the particles by a finite random walk. At each time step three independent random components of displacement are generated for each particle from the corresponding Gaussian distribution. The r.m.s. displacement $\Delta x$ in any direction of an $i$-fold particle is

$$
\Delta x_{i}=\left(2 D_{i} \Delta t\right)^{\frac{1}{2}}
$$


where $D_{i}=K_{\mathrm{B}} / 6 \pi r_{i}$, is the particle diffusivity. $D_{i}$ can be obtained in terms of the diffusivity $D_{0}$ of an elemental particle by

$$
D_{i}=D_{0} i^{-\frac{1}{3}}
$$

Particle collisions are simulated on the basis of straight-line trajectories during each time step. The question therefore arises of the validity of this as an approximation to Brownian-induced coagulation. The r.m.s. displacement has been chosen correctly, but a particle of mass $m$ undergoing Brownian motion actually travels along a tortuous path at r.m.s. speed $(k T / m)^{\frac{1}{2}}$. At first sight this suggests that the simulation would underpredict the collision rate. However, replacing Brownian motion by a finite random walk must change the pair-distribution function, that is to say the probability distribution function for the spacing between any given pair of particles. So, while modelling Brownian motion by a finite random walk introduces inefficiency into the basic collision process, it can compensate by increasing the probability that any pair of particles are found close together at the beginning of a time step. Here 'close together' means a separation on the scale of the r.m.s. steplength of the random walk. These matters are investigated in detail in the Appendix. Tests with the non-coagulating form of the program have shown that satisfactory collision rates for monodisperse populations of particles are obtained when the ratio $\Delta x / r$ is about 0.5 . It is important to use the maximum possible time step in order to minimize computation times.

\subsection{Laminar shear}

The coagulating effects of a velocity gradient are investigated by imposing a uniform shearing motion on the control volume:

$$
u_{1}=g x_{3},
$$

with $G$ the shear rate. The particles are assumed to move with the fluid so their displacement in any time step is just

$$
Y(i)=\left(Y_{1}(i), 0,0\right), \quad Y_{1}(i)=G P_{3}(i) \Delta t .
$$

As stated in $\$ 1$, in this paper we are ignoring hydrodynamic interactions between particles. The large body of work on particle interactions in low-Reynolds-number flows (for a review see e.g. Mason 1976) shows that hydrodynamic forces will always come into play in a detailed analysis of collision dynamics. This is investigated in detail in Part 2 (Valioulis, List \& Pearson 1984).

A uniform shearing motion, on average, moves a fraction of the particles out of the control volume at every time step. If they were replaced in the control volume according to simple periodic boundary conditions $\left(P_{1}=P_{1}-L\right.$, whenever $\left.P_{1}>L\right)$ the simulation would be completely deterministic once initial positions had been chosen for the particles. Each particle would move in a straight line with fixed $P_{2}$ and $P_{3}$ coordinates. After a certain time all collisions between existing particles would cease, as each particle would have swept out its own track through the control volume. In a real flow this would not occur, as particles are continually meeting 'new' particles. Therefore, in the simulation, when a particle leaves the volume it is replaced at randomly chosen height $P_{3}$ on the other side of the control volume. The random value of the height $P_{3}$ must be chosen from a distribution that reflects the increasing flux of particles at larger values of $P_{3}$. This flux is proportional to $P_{3}$, and a uniformly distributed random variate may be converted to this linear p.d.f. by taking its square root. This strategy leads to a further complication: particles may be replaced on top 
of one another, leading to spurious collisions. This is almost totally eliminated by checking for such particle overlaps at the end of each time step and randomly moving one of each overlapping pair. This may introduce a few further overlaps as no final check is made. An estimate of this number is available from the number of initial overlaps, which is recorded. This error is acceptable in the light of other approximations in the simulation. Overlaps are also introduced by the process of adding new elemental particles at each time step, whatever the collision mechanism. All types of overlaps are resolved simultaneously in the same manner.

\subsection{Turbulent shear}

We wish to simulate the coagulation of small particles by turbulent flow. The motion of a suspended particle can be identified with the motion of an adjacent fluid particle provided that the timescale of the (fluid) particle acceleration is much greater than the particle relaxation time $t_{\mathbf{r}}$; that is to say, if inertial effects are negligible, as will be the case here. Then for particles of radius smaller than the smallest scale of the turbulent motion (the Kolmogorov lengthscale $\left(\left(\nu^{3} / \epsilon\right)^{\frac{1}{2}}\right)$, coagulation rates are determined solely by the kinematics of the small scales of the turbulent flow field, in particular by the r.m.s. strain rate $(\epsilon / \nu)^{\frac{1}{2}} / 15^{\frac{1}{2}}$. These small scales are very nearly isotropic (Batchelor 1953).

Under these conditions, two particles separated by a distance smaller than the Kolmogorov lengthscale are subjected to a motion that can be decomposed into a rigid-body rotation representing the local vorticity, and a locally uniform threedimensional straining motion. The rigid-body-rotation component of the motion has no effect on the collisions of non-interacting particles, and so only the straining motion (with symmetric velocity-gradient tensor) is modelled. The straining motion will be uniform over lengthscales smaller than the Kolmogorov microscale, but there is no agreement as to the duration of this straining (Monin \& Yaglom 1975). Two timescales are important for the small-scale straining: the rate of rotation of the principal axes of strain and the rate of change of the magnitude of the principal rates of strain. For turbulent flow at high Reynolds number the rate of change of the deformation fields of the small eddies is related to the Langrangian time microscale $\alpha$ (Lumley 1972). The timescale of the deformation field is $\lambda / u^{\prime}$, where $\lambda$ is the Taylor microscale and $u^{\prime}$ the r.m.s. fluctuating velocity. Corrsin (1963) approximates the ratio of the two as

$$
\alpha u^{\prime} / \lambda \approx\left(\frac{1}{30} R_{\lambda}\right)^{\frac{1}{2}}
$$

and since by definition

we have

$$
\lambda^{2}=15 \nu \frac{\overline{u^{\prime 2}}}{\epsilon}, \quad R_{\lambda}=\frac{u^{\prime} \lambda}{\nu}
$$

$$
\frac{\alpha}{(\nu / \epsilon)^{\frac{1}{2}}} \approx\left(\frac{1}{2} R_{\lambda}\right)^{\frac{1}{2}}
$$

which implies that the strain and vorticity of the small eddies remain constant for a time interval at least equal to the Kolmogorov timescale $t=(\nu / \epsilon)^{\frac{1}{2}}$. This is just the inverse of the characteristic strain rate.

The effect of the rate of rotation of the principal axes of strain on the collision rate was investigated using the monodisperse, non-coagulating version of the simulation. The velocity gradient was simulated so that both the principal axes and principal rates of strain could be changed independently. The magnitude of the strain was kept constant for a time interval equal to the Kolmogorov timescale. No statistically 
significant difference in the collision rate was found, whatever the timescale of rotation of the principal axes of strain. Therefore in the coagulation simulation both principal axes and rates of strain were varied at the same rate.

Assuming homogeneous, isotropic, unbounded turbulence with a Gaussian velocity gradient field, the elements of the rate-of-strain tensor were chosen randomly to satisfy (Hinze 1959)

$$
\frac{\partial u_{i}}{\partial x_{k}} \frac{\partial u_{j}}{\partial x_{l}}=\left\{\begin{array}{l}
\frac{1}{15} \frac{\epsilon}{\nu} \quad(i=j=k=l), \\
-\frac{1}{30} \frac{\epsilon}{\nu} \quad(j=l \text { and } i=k, \text { or } i=l \text { and } j=k, \text { and } i \neq j), \\
\frac{2}{15} \frac{\epsilon}{\nu} \quad(k=l \text { and } i=j \text { and } i \neq k), \\
0 \quad \text { (all other combinations), }
\end{array}\right.
$$

subject to

$$
\frac{\partial u_{i}}{\partial x_{i}}=0
$$

and kept constant for a time interval equal to the Kolmogorov timescale.

The simulation proceeds as in the case of laminar shear with particle displacements being given by the product of the time step $\Delta t$ and the fluid velocity corresponding to the particle position. Now, however, as the motion is three-dimensional and stochastic, true periodic boundary conditions can be used. This corresponds to the control volume being surrounded by copies which are deformed with the original. Particles in the control volume at the end of one time step can then be used for the next. However, in preliminary simulations, random fluctuations in the number of particles were found to cause trouble. To avoid the program halting because of too many or no particles left in the control volume the total number was adjusted at each time step according to

$$
N_{i+1}=N_{i}-N_{\text {col }}+N_{\mathrm{c}}
$$

where $N_{\text {col }}$ is the number of collisions that had occurred during the time step, and $N_{c}$ the number of elemental particles added. In order to satisfy the above condition, either particles were removed at random, or a particle whose volume had been chosen at random from the existing population was added at a random position. Finally, particle overlaps were resolved as explained in $\S 3.4$.

\subsection{Multiple mechanisms}

Simulations were performed in which the particle displacement was the linear sum of a fluid shearing and a Brownian component. The relative magnitude of the Brownian and shearing parameters could then be varied to investigate their interaction.

\section{Results}

Figure 3 shows the effect of changing the r.m.s. steplength on collision rate in Brownian motion (see the Appendix for a discussion). There is some statistical scatter in the results, but the general shape of the curve is correct. From these results a suitable time step can be chosen for simulations involving Brownian motion. Similar computations of collision rates in laminar- and turbulent-shear-induced coagulation 


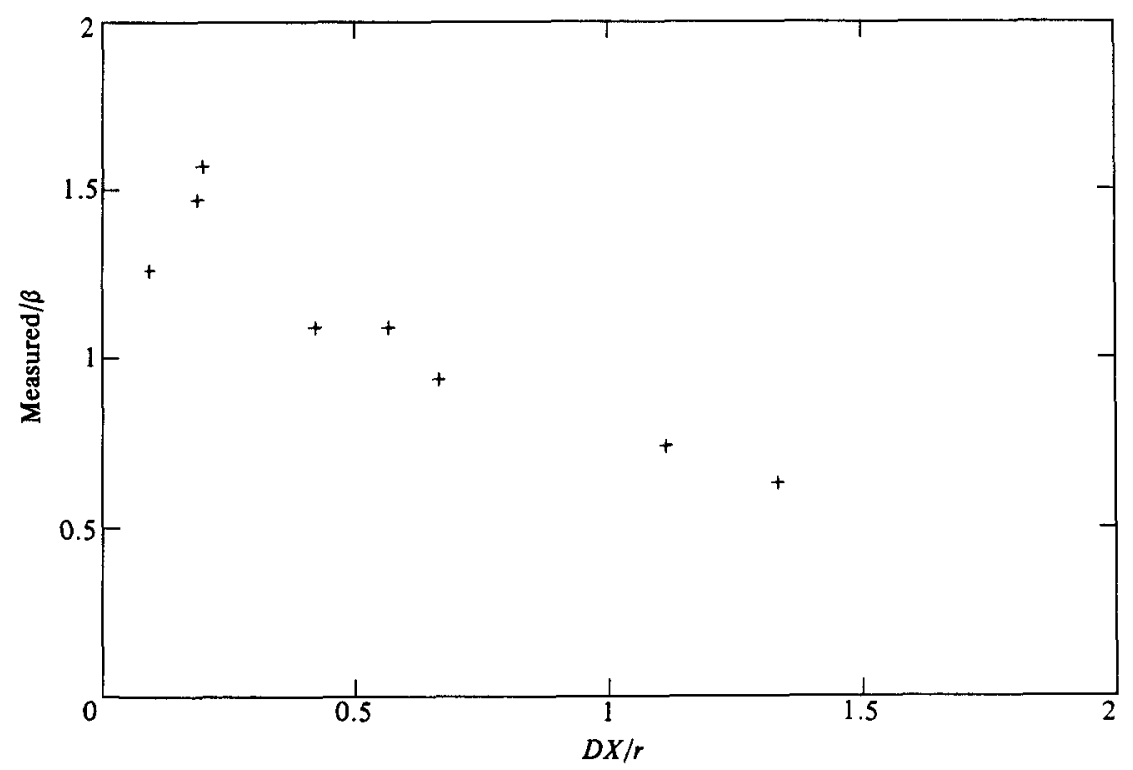

Figure 3. Simulated collision rate of monodisperse particles undergoing Brownian motion as a function of r.m.s. displacement. The ratio between measured collision rate and theoretical rate is plotted against the ratio of steplength to particle radius.

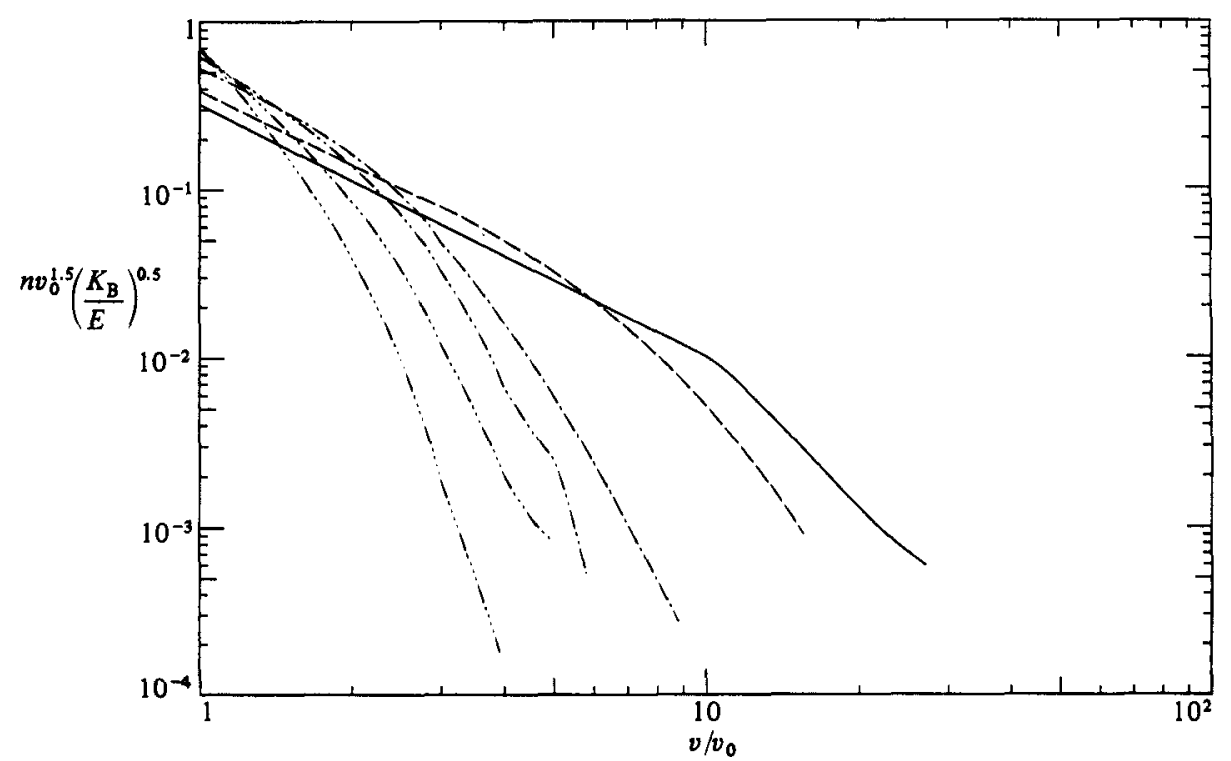

FIgure 4. Development towards steady state of size distribution of initially monodisperse population undergoing Brownian-induced coagulation; $D_{0}=0.222, E=5.6 \times 10^{-5}, i_{\max }=125$ :

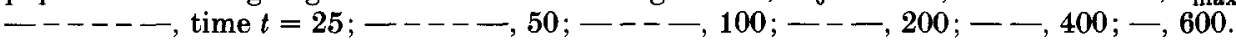

were performed to check that they yielded the values given by table 1 . This, indeed, was found to be the case. The result for turbulent shear due to Saffman \& Turner (1956) has been amended by a factor of $\pi^{\frac{1}{2}}$ from that in the original paper, correcting an algebraic error.

The development of a size distribution in a typical simulation starting with particles all of unit volume $v_{0}$ and undergoing Brownian-induced coagulation is shown 


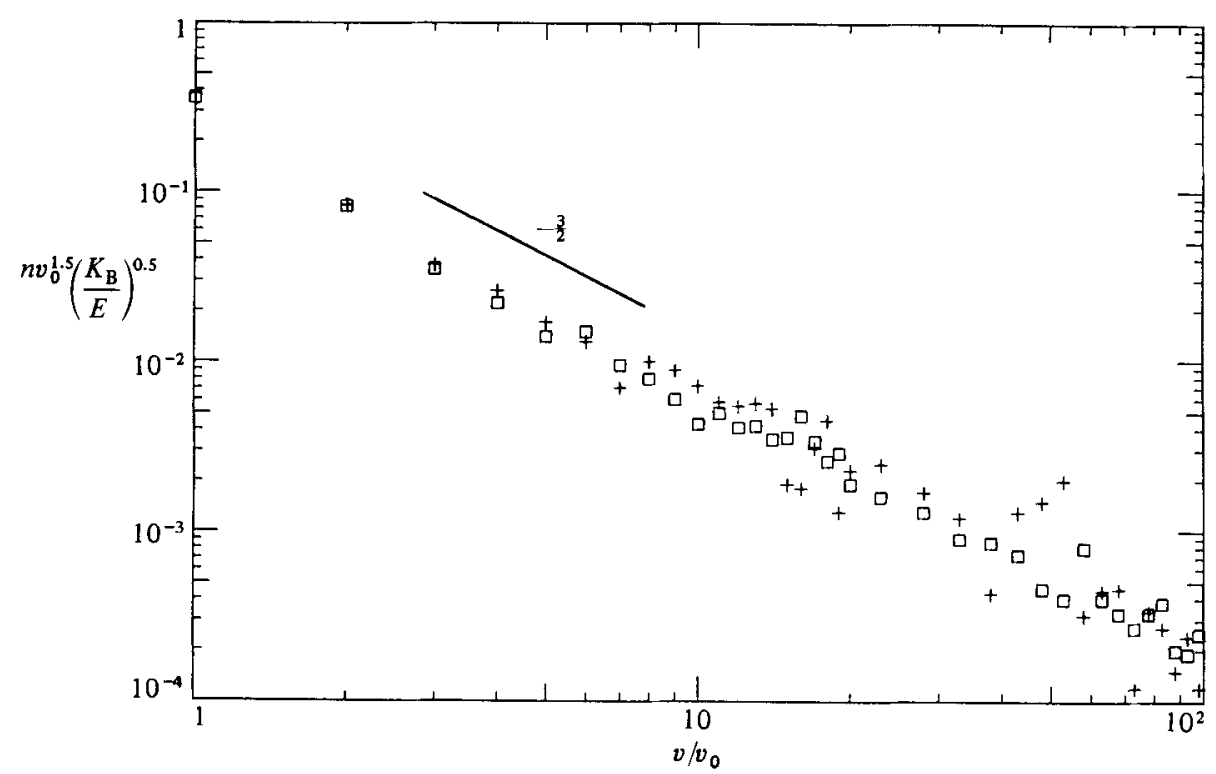

Figure 5. Steady-state non-dimensional size distribution for Brownian motion; $D_{0}=0.222$, $i_{\max }=125:+, E=5.6 \times 10^{-5}, \phi=0.016 ; \square, E=4.4 \times 10^{-4}, \phi=0.043$.

in figure 4. The size distribution is non-dimensionalized according to (3) and plotted logarithmically against particle volume non-dimensionalized with the unit particle volume. The curves plotted are smoothed approximations to the actual data points, at $v=i v_{0}$, which are rather scattered. The upper portion of the data attains a slope of $-\frac{3}{2}$ once a range of about one decade in volume has been reached. Then, as particles of increasing size are formed, the slope of the size distribution remains the same, but its absolute level declines gradually. It reaches a statistically steady state once the first large particle is lost from the system. The final steady state for this set of parameters is shown in figure 5, along with that for a run at a higher final volume concentration $\phi$ (this is obtained by adding more particles at each time step). The points plotted are actual data from the simulations, averaged over 1000 time steps. Even with this time averaging, there is still some statistical scatter in the data, especially at the lower end of the size distribution where very small numbers of particles are actually involved. To smooth the data further in the region $v / v_{0}=20-100$, they have been averaged in groups of 5 .

For both these runs $v_{\max }=125 v_{0}$, although the volume distribution is only plotted out to $v / v_{0}=100$. Beyond this the data becomes erratic. The two sets of data are fully collapsed by the normalization used and very clearly exhibit the $-\frac{3}{2}$ power law expected from Hunt's (1982) theory. The intercept of the best-fit line of slope $-\frac{3}{2}$ with the axis $v / v_{0}=1$ gives the constant $a_{\mathrm{B}}$ in (3).

Figure 6 is a comparison of the steady-state size distributions for laminar shear at two volume concentrations differing by an order of magnitude. Again the data points are averaged over 1000 time steps, and are collapsed onto a slope of -2 by the normalization suggested by dimensional arguments. Similar results are shown for turbulent shear in figure 7, where the inverse of the Kolmogorov timescale, $(\epsilon / \nu)^{\frac{1}{2}}$, is used in place of $G$ in the normalization of the size distribution. Again, a -2 power law is achieved at steady state, and the normalized results are independent of the flux of particle volume through the size range. Note, however, that the data points 


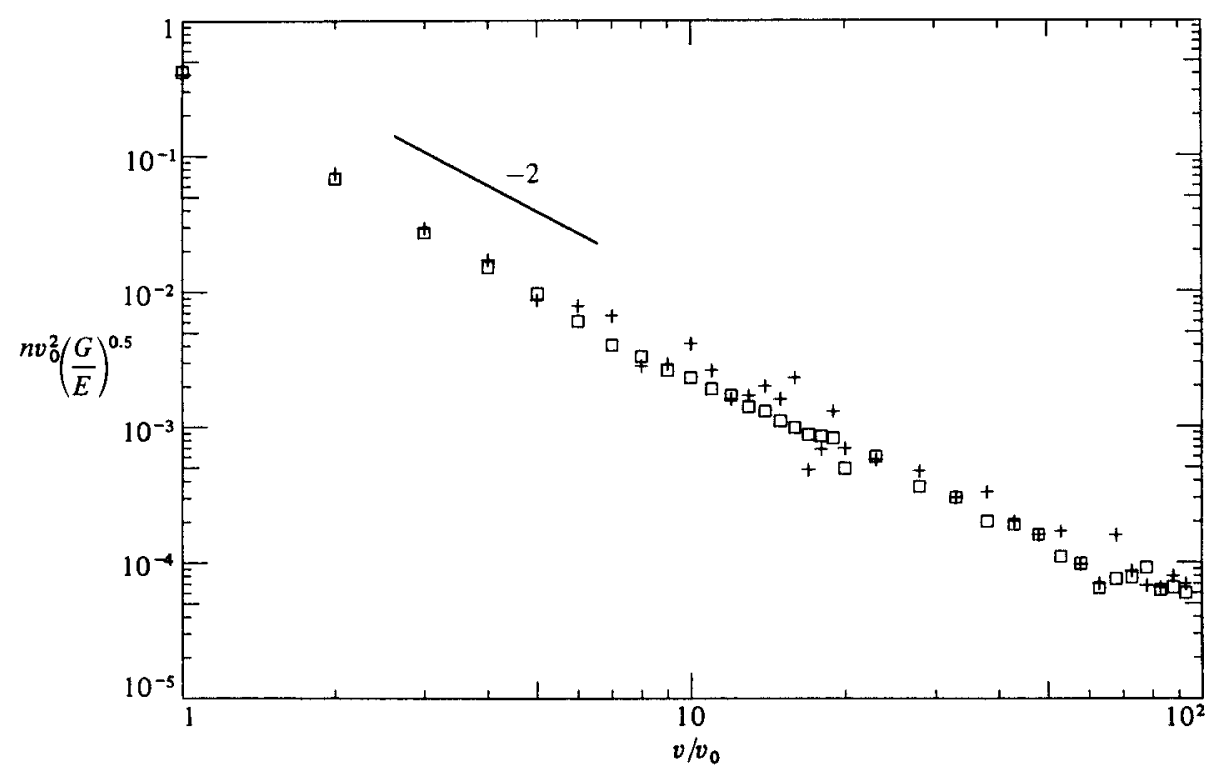

FIGURE 6. Steady-state non-dimensional size distribution for laminar shear:,$+ G=0.25$, $E=1.4 \times 10^{-5}, i_{\max }=125, \phi=0.013 ; \square, G=1, E=1.1 \times 10^{-3}, i_{\max }=125, \phi=0.114$.

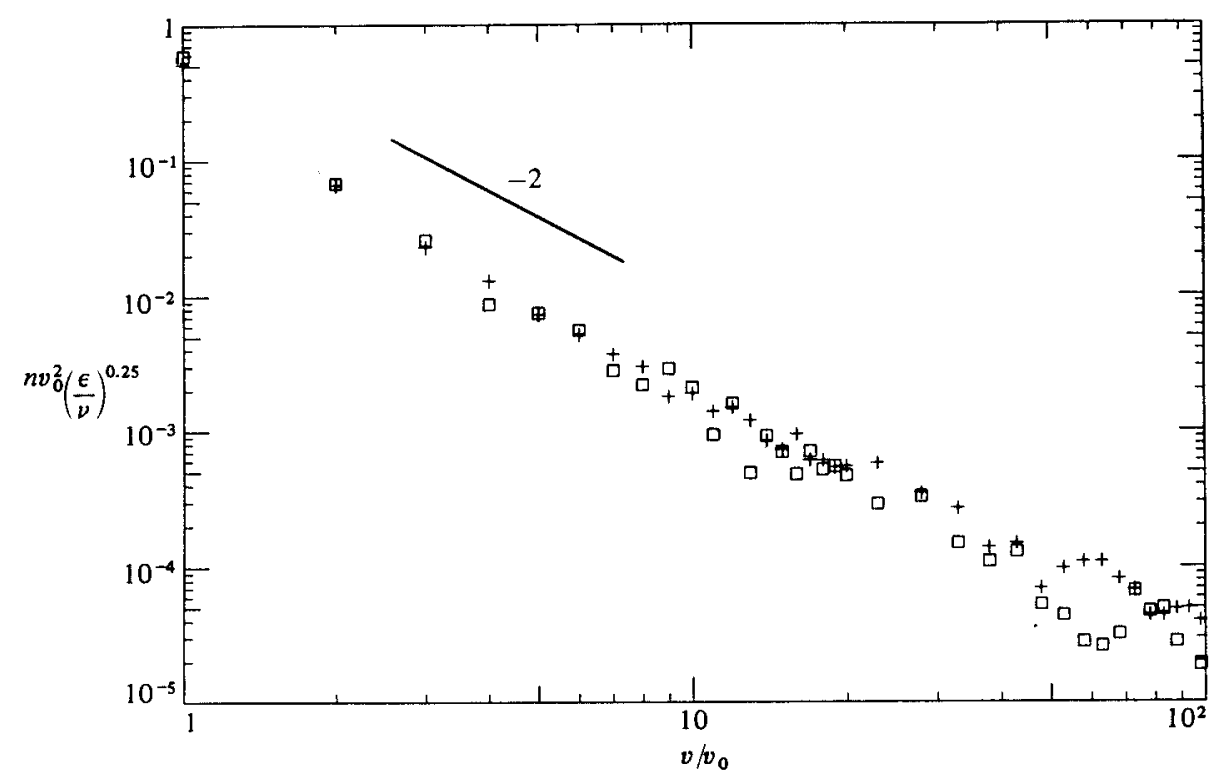

FigURE 7. Steady-state non-dimensional size distribution for turbulent shear; $K_{\mathrm{sh}}=0.5$, $i_{\max }=125:+, E=5.7 \times 10^{-4}, \phi=0.053 ; \square, E=1.4 \times 10^{-5}, \phi=0.008$.

are slightly lower than in the case of laminar shear. This is simply a consequence of the collision functions given in table 1: the expressions for laminar and isotropic turbulent shear are identical if $G$ is replaced by $1.72(\epsilon / \nu)^{\frac{1}{2}}$. With this scaling the data of figures 6 and 7 collapse. This result strongly suggests the equivalence of laminar rectilinear shear and three-dimensional turbulent shear as coagulating agents. It is gratifying that the results of the simulations, which do not assume forms for the 


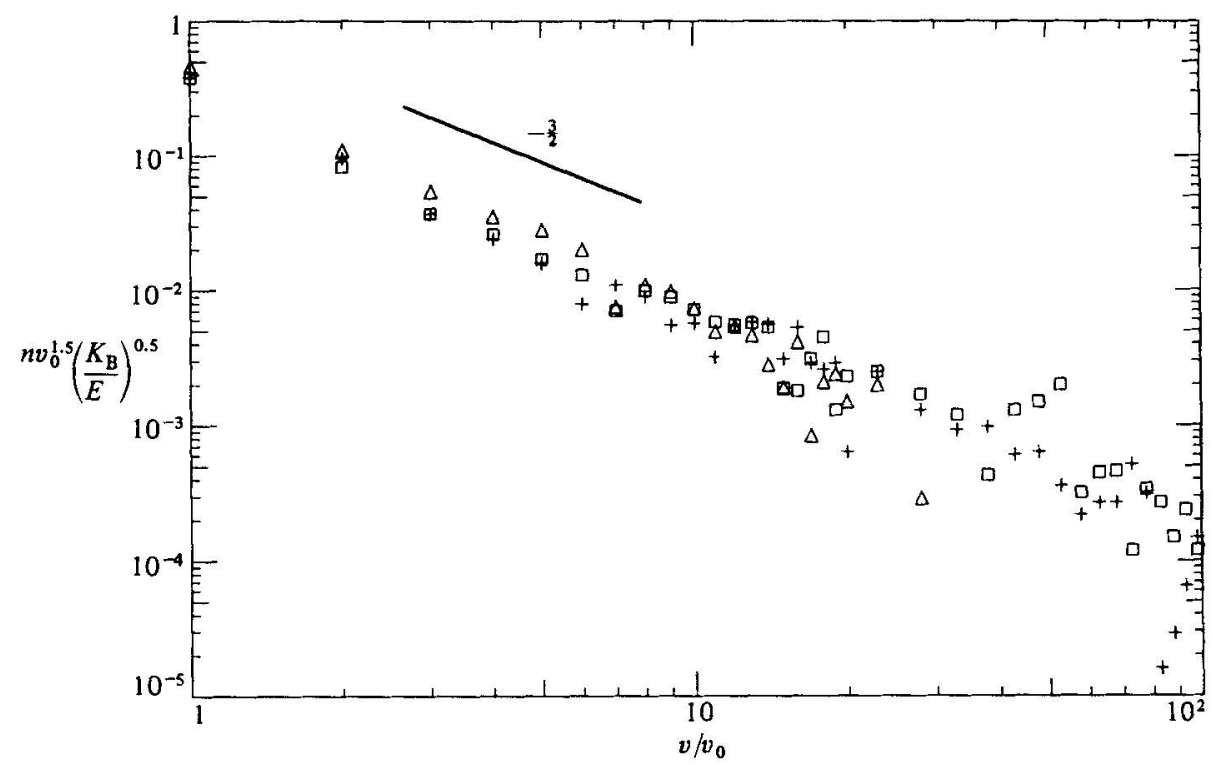

Figure 8. Comparison of steady-state non-dimensional size distribution for Brownian motion for different $i_{\max }: D_{0}=0.222, E=5.6 \times 10^{-5} ; \triangle, i_{\max }=27, \phi=0.011 ;+, i_{\max }=125, \phi=0.016$; ㅁ. $i_{\max }=512, \phi=0.0022$.

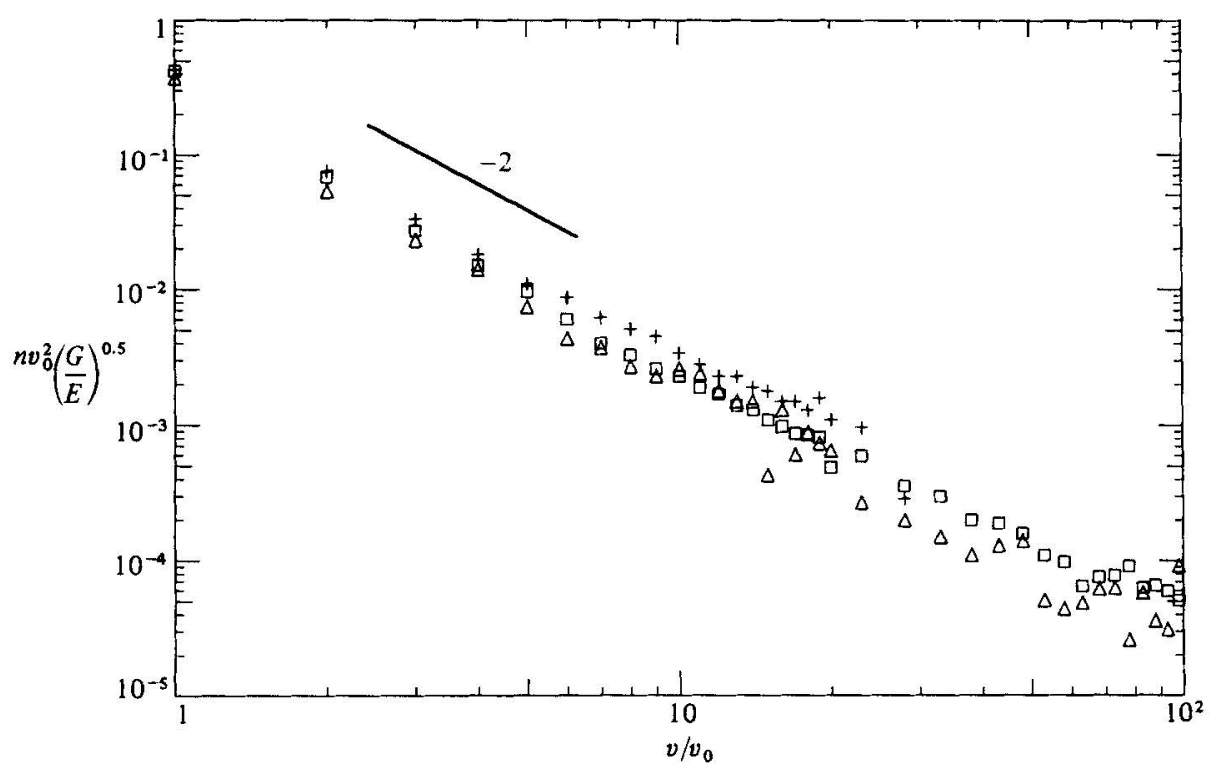

Figure 9. Comparison of steady-state non-dimensional size distribution for laminar shear for different $i_{\max }:+, G=1, E=1.1 \times 10^{-3}, i_{\max }=27, \phi=0.049 ; \square, 1,1.1 \times 10^{-3}, 125,0.057 ; \triangle, 0.25$, $1.4 \times 10^{-6}, 512,0.014$

collision functions $\beta$, agree well with arguments suggested by the analytic estimates for $\beta$.

The next series of simulation runs illustrate the effect that the ratio $i_{\max }=v_{\max } / v_{0}$ (i.e. the size range covered by the simulation has on final steady-state size distributions in Brownian motion and laminar shear. Figures 8 and 9 give size 


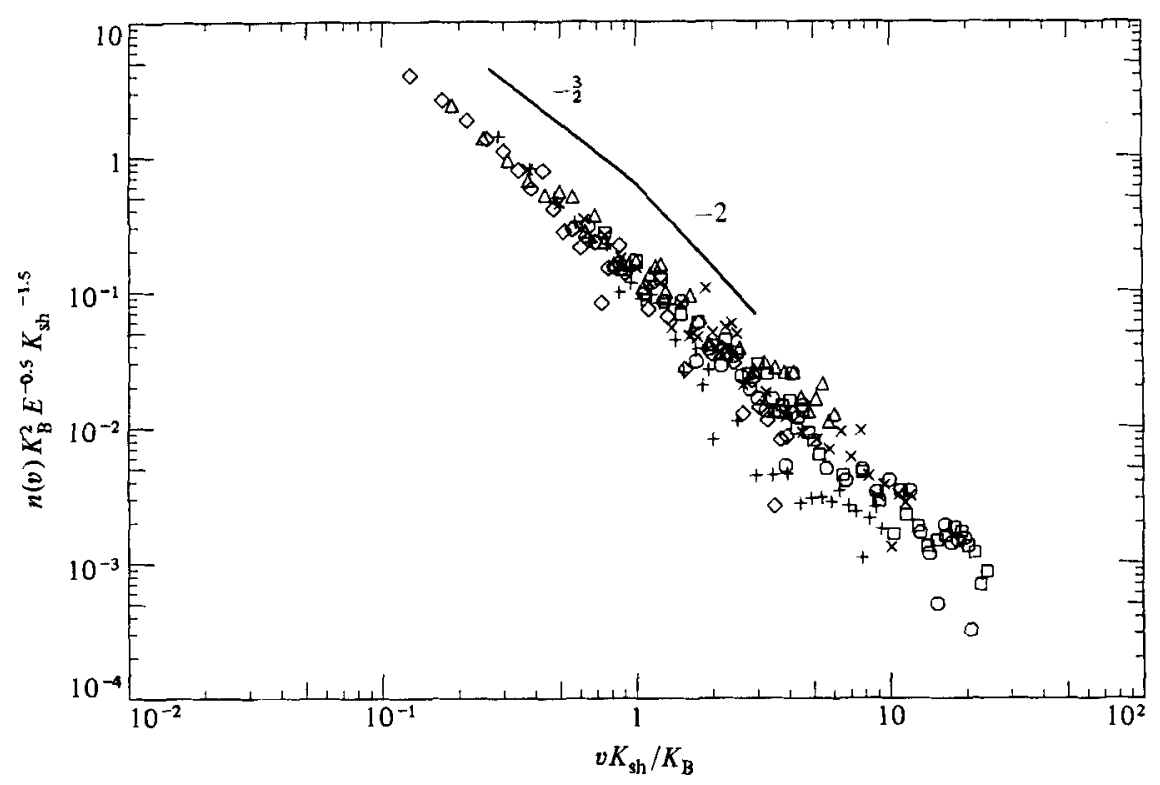

FIGURE 10. Steady-state normalized size distribution for (i) Brownian motion and laminar shear, and (ii) Brownian motion and turbulent shear. (i) $E=5.6 \times 10^{-5}, i_{\max }=125: \square, K_{\mathrm{sh}}=0.25$, $\phi=0.0081 ; \times, 0.125,0.011 ; \triangle, 0.063,0.013$. (ii) $+, K_{\mathrm{sh}}=0.344, \mathrm{D}_{0}=0.8, \mathrm{E}=1.7 \times 10^{-5}$, $\mathrm{i}_{\max }=512, \phi=0.0096 ; \diamond, 0.215,0.222,5.6 \times 10^{-5}, 125,0.0077 ; 0,0.043,0.222,5.6 \times 10^{-5}, 125$, 0.013 .

distributions for the three cases $v_{\max } / v_{0}=27,125$ and 512. In all cases the relevant $-\frac{3}{2}$ or -2 power law prevails. For Brownian motion the results for $v_{\max } / v_{0}=125$ and 512 are indistinguishable, while those for the smallest size range are slightly higher at the upper end of the size range. For laminar shear there is a slight but consistent decline in level with increasing size range. This reflects the extent to which the size distribution is affected by the collisions of the relatively small number of large particles. In laminar shear the collision function increased with the volume of the particles involved faster than in Brownian coagulation. Work on the effects of hydrodynamic interactions between particles on coagulation (for the most recent study see Adler 1981) suggests that they act to reduce most the collision rate between particles of widely different sizes. This would probably result in weaker dependence of the level of the size distribution (the value of $a_{\mathrm{sh}}$ ) on the size range covered by the-simulation. Further work, with a more sophisticated simulation incorporating hydrodynamic interactions, will elucidate this point.

A consensus of the simulations performed gives the values

$$
a_{\mathrm{B}}=0.2 \pm 0.04, \quad a_{\mathrm{sh}}=0.24 \pm 0.05 \text {. }
$$

However, it is likely that accounting for hydrodynamic and interfacial forces will alter the values of these dimensionless constants.

So far all the results have been for simulations in which only one collision mechanism has been present. We now turn to cases where both Brownian motion and fluid shearing operate. A new normalization of the size distribution and volume variable is now required to collapse all the data. Following Hunt (1982) we define a non-dimensional volume

$$
\chi=v K_{\mathrm{sh}} / K_{\mathrm{B}}
$$


where $K_{\mathrm{sh}}$ represents $G$ or $1.72(\epsilon / \nu)^{\frac{1}{2}}$, and $K_{\mathrm{B}}$ is as before. This is such that the collision rates due to Brownian motion and shear are equal for particles of size $\chi \approx 1$. Then if a normalized size distribution is defined by

(3) and (4) reduce to

$$
n^{*}=n\left(\frac{K_{\mathrm{B}}}{K_{\mathrm{sh}}}\right)^{2}\left(\frac{K_{\mathrm{sh}}}{E}\right)^{\frac{1}{2}}
$$

$$
n^{*}(\chi)=a_{\mathrm{B}} \chi^{-\frac{3}{2}} \text { and } n^{*}(\chi)=a_{\mathrm{sh}} \chi^{-2} .
$$

Results of three simulations each for laminar and turbulent shear with Brownian motion are plotted in this normalized form in figure 10. Lines of slope $-\frac{3}{2}$ and -2 are drawn for comparison. There is some indication of a change in slope around $\chi=1$ but it is not conclusive. Also, the constants $a_{\mathrm{B}}$ and $a_{\mathrm{sh}}$ obtained from the data in figure 10 are the same (within statistical error) as those obtained from simulations with only one collision mechanism present, providing some support for the hypothesis of non-interference of mechanisms.

\section{Discussion}

The main aims of this study have been:

1. to study the feasibility of a Monte Carlo simulation of both the collision function $\beta$ and the coagulation equation (2) for the evolution of a population of particles to a steady state;

2. the investigation of Hunt's (1982) theory for the form of the resulting size distributions.

The simulation method described has proved most successful in modelling the coagulating powers of both Brownian and bulk shearing mechanisms and the development of steady-state size distributions. This is in spite of the relatively restricted range of particle sizes that can be followed in any one computer run and the somewhat artificial strategy of adding new unit particles at each time step.

The results show that final steady state is rather insensitive to the size range covered, and that the size distribution at the upper end (small particles) is not very disturbed by replacing the interactions of all small particles with the addition of unit particles at a constant rate. These observations are in accord with the striking success of dimensional analysis in predicting the observed size distributions. For dimensional analysis to be successful the dynamics of the coagulation process must be mainly 'local' in size space so that further independent parameters (such as $v_{0}$ and $v_{\text {max }}$ ) are not important. We expect that accounting for hydrodynamic interactions between particles will decrease the dependence of the level of the size distribution, for given volume flux, in shear-induced coagulation. Notice that the evolving populations of particles start to exhibit the relevant power law over much of their size distribution long before a steady state is reached.

Hunt's further hypothesis that different collision mechanisms can act independently over separate size ranges has been partially confirmed. A slope of $-\frac{3}{2}$ is not very different from one -2 when there is scatter in the data! However, complete resolution of this point would require the simulation to cover a greater range of particle sizes. This is not feasible with the available computer storage. The perturbation analysis of van de Ven \& Mason (1977), for the effect of weak shear on Brownian coagulation, suggests that when hydrodynamic interactions are considered the two mechanisms may not be strictly additive.

In conclusion, it ean be said that, while simple in concept, and using acceptable 
computer resources, the simulation method has provided useful elucidation of Hunt's hypotheses and experimental results under carefully controlled conditions. Further work on the technique to include hydrodynamic interactions, interfacial forces and gravitational settling is reported in Part 2 (Valioulis et al. 1984).

Financial support for this work was provided by N0AA/Sea Grant NA80AA-D-00120, N0AA Grant NA80RA-D0-0084 and a Mellon Foundation Grant to the Environmental Quality Laboratory at Caltech. The authors would also like to thank Dr R. C. Y. Koh for advice on many aspects of the computer code and Dr R. C. Flagan for helpful discussions of the problem.

\section{Appendix. Finite steplength and collision rate in Brownian motion}

The theoretical collision function $\beta$ for Brownian-induced collisions between particles of radii $r_{i}$ and $r_{j}$ given in table 1 was computed (see e.g. Chandrasekhar 1949) by solving a diffusion equation for the pair-distribution function $w(s)$, where $s$ is the distance beteen the particles. In particular, the collision function is given by the asymptotic flux to the surface of a fixed sphere of radius $\sigma=r_{i}+r_{j}$, with a total diffusivity $D=D_{i}+D_{j}$. The 'concentration' $w$ is held at zero at $s=\sigma$ and at unity at $s=\infty$. Initially $w$ is uniform outside the sphere. Then at large times the pairdistribution function is given by

$$
w=1-\frac{\sigma}{s}
$$

whence the required result

$$
\beta=4 \pi D\left(s^{2} \frac{\mathrm{d} w}{\mathrm{~d} s}\right)_{s=\sigma}=4 \pi D \sigma
$$

If the actual pair-distribution function in the finite-steplength simulation was identical with that in (A 1) then the collision rate measured would be no larger than one-half of that in (A 2), however small the steplength. This result can be obtained either by careful evaluation of the expected collision probability from the algorithms used for generating particle displacements and detecting collisions, or by the following simple argument. In the limit of $\Delta x \ll \sigma$, i.e. very small r.m.s. steplength, but still with $\Delta t \gg t_{\mathrm{r}}$, two particles must be so close at the beginning of the time step in which they collide that the curvature of their surfaces may be neglected. The problem then reduces to that of the collision of a diffusing point with an adsorbing plane and we need only consider the component of the random walk perpendicular to the plane.

Consider now this one-dimensional problem. The particle is judged to have collided with the plane if its final position is on the far side of the plane. For any given final position on the far side of the plane there is a whole class of possible Brownian trajectories leading to it. Now each of these trajectories must cross the plane for the first time at some point. There will be an associated trajectory defined to be identical with the original until the first contact with the adsorbing plane and then the mirror image, in the plane, of the original. As the endpoint of this associated trajectory lies on the near side of the plane it would not be judged a collision by the collision algorithm. Hence the $50 \%$ inefficiency.

However, for the same reason, the pair-distribution function will not be identical in the theoretical and simulated cases. In the finite-steplength case, $w$ will be larger within a distance of order $\Delta x$ of $s=\sigma$. This can compensate for the basic inefficiency of the collision algorithm. The actual form of $w$ for a given distribution of steplengths and hence the collision function could be computed by solving the relevant integral equation. This has not been done as yet, but the non-coagulating form of the 
simulation has been used to determine the collision rate for a monodisperse population of particles as a function of the mean steplength. The results of this 'experimental' determination are shown in figure 3 . The ratio of measured collision rate to that predicted from (A 2) is plotted against the ratio of r.m.s. displacement $\Delta x$ in any direction, and the particle radius $r$. The ratio is unity for $\Delta x / r$ about 0.6 , and so $\Delta x$ is chosen accordingly in all the coagulation simulations.

\section{REFERENCES}

Abramowitz, M. \& Stegun, I. A. 1972 Handbook of Mathemtical Functions. Natl Bur. Stand. AdLer, P. M. 1981 Heterocoagulation in shear flow. J. Coll. Interface Sci. 83, 106-115.

Alder, B. J. \& Wainwright, T. E. 1959 Studies in molecular dynamics. I. General method. J. Chem. Phys. 31, 459-466.

Batchelon, G. K. 1953 The Theory of Homogeneous Turbulence. Cambridge University Press.

Chandrasekhar, S. 1943 Stochastic problems in physics and astronomy. Rev. Mod. Phys. 15, 1-89.

Corrsin, S. 1963 Estimates of the relations between Eulerian and Lagrangian scale in large Reynolds number turbulence. J. Atmos. Sci. 20, 115.

Findheisen, W. 1939 Meteor. Z. 56, 356.

Friedlander, S. K. $1960 a$ On the particle size spectrum of atmospheric aerosols. J. Meteor. 17, 373-374.

Friedlander, S. K. $1960 b$ Similarity considerations for the particle-size spectrum of a coagulating, sedimenting aerosol. J. Meteor. 17, 479-483.

Gartrell, G. \& Friedlander, S. K. 1975 Relating particulate pollution to sources: the 1972 California aerosol characterization study. Atmos. Environ. 9, 279-294.

Gelbard, F., Tambovr, Y. \& Seinfeld, J. H. 1980 Sectional representations for simulating aerosol dynamics. J. Coll. Interface Sci. 76, 541-556.

Gillespie, D. T. 1972 The stochastic coalescence model for cloud droplet growth. J. Atmos. Sci. 29, 1496-1510.

Hinze, J. O. 1975 Turbulence, 2nd edn. MeGraw-Hill.

HUnT, J. R. 1982 Self-similar particle size distributions during coagulations: theory and experimental verification. J. Fluid Mech. 122, 169-185.

Husar, R. B. 1971 Coagulation of Knudsen aerosols. Ph.D. thesis, The University of Minnesota, Minneapolis.

LUMLEY, J. L. 1972 On the solution of equations describing small scale deformation. In Symposia Mathematica: Convegno sulla Teoria della Turbolenza al Instituto de Alta Matematica. Academic.

Mason, S. G. 1977 Orthokinetic phenomena in disperse systems. J. Coll. Interface Sci. 58, 275-285.

Monin, A. S. \& Yaglom, A. M. 1975 Statistical Fluid Mechanics, vol. 2. MIT Press.

Nowakowski, R. \& Sitarski, M. 1981 Brownian coagulation of aerosol particles by Monte Carlo simulation. J. Coll Interface Sci. 83, 614-622.

Pruppacher, H. R. \& KLetT, J. D. 1978 Microphysics of Clouds and Precipitation. Reidel.

Saffman, P. G. \& Turner, J. S. 1956 On the collision of drops in turbulent clouds. J. Fluid Mech. 1, 16-30.

Sмоцбсноwsкi, M. 1916 Drei Vorträge über Diffusion brownsche Bewegung und Koagulation von Kolloidteilehen. Physik Z. 17, 557-585.

SмоцUсношsкi, M. 1917 Versuch einer mathematischen Theorie der Koagulationskinetic kolloider Lösungen. Z. Phys. Chem. 92, 129.

Valtoulis, I. A., List, E. J. \& Pkarson, H. J. 1984 Monte Carlo simulation of coagulation in discrete particle-size distributions. Part 2. Interparticle forces and the quasi-stationary equilibrium hypothesis. J. Fluid Mech. 143, 387-411.

VAN DE VEN, T. G. M. \& MASON, S. G. 1977 The microrheology of colloidal dispersions. VIII. Effect of shear on perikinetic doublet formation. Coll. Polymer Sci. 255, 794-804.

Zeichner, G. R. \& Schowalter, W. R. 1977 Use of trajectory analysis to study the stability of colloidal dispersions in flow fields. AIChE J. 23, 243-254. 